\title{
Sparse DSI: Learning DSI Structure for Denoising and Fast Imaging
}

\author{
Alexandre Gramfort ${ }^{1,2}$, Cyril Poupon ${ }^{2}$, and Maxime Descoteaux ${ }^{3}$ \\ 1 Parietal Team, INRIA Saclay-île-de-France, Saclay, France \\ alexandre.gramfort@inria.fr \\ 2 CEA, DSV, I²BM, Neurospin bât 145, 91191 Gif-Sur-Yvette, France \\ 3 SCIL, Computer Science department, Université de Sherbrooke
}

\begin{abstract}
Diffusion spectrum imaging (DSI) from multiple diffusionweighted images (DWI) allows to image the complex geometry of water diffusion in biological tissue. To capture the structure of DSI data, we propose to use sparse coding constrained by physical properties of the signal, namely symmetry and positivity, to learn a dictionary of diffusion profiles. Given this estimated model of the signal, we can extract better estimates of the signal from noisy measurements and also speed up acquisition by reducing the number of acquired DWI while giving access to high resolution DSI data. The method learns jointly for all the acquired DWI and scales to full brain data. Working with two sets of 515 DWI images acquired on two different subjects we show that using just half of the data (258 DWI) we can better predict the other $257 \mathrm{DWI}$ than the classic symmetry procedure. The observation holds even if the diffusion profiles are estimated on a different subject dataset from an undersampled q-space of 40 measurements.
\end{abstract}

\section{Introduction}

Diffusion-weighted imaging offers a way to non-invasively image the diffusion of water molecules in biological tissue. The ensemble average propagator (EAP) formalism provides a powerful framework to describe and predict the diffusion behavior of water molecules in complex materials. Under the narrow pulse assumption, there is a Fourier relationship between the measured DWI signal and diffusion propagator, $P(\mathbf{R})$,

$$
P(\mathbf{R})=\int_{\mathbf{q} \in \mathbb{R}^{3}} E(\mathbf{q}) e^{-2 \pi i \mathbf{q} \cdot \mathbf{R}} d \mathbf{q},
$$

with $E(\mathbf{q})=S(\mathbf{q}) / S_{0}$, where $S(\mathbf{q})$ is the diffusion signal measured at position $\mathbf{q}$ in q-space, and $S_{0}$ is the baseline image acquired without any diffusion sensitization $(q=0)$. We denote $q=|\mathbf{q}|$ and $\mathbf{q}=q \mathbf{u}, \mathbf{R}=r \mathbf{r}$, where $\mathbf{u}$ and $\mathbf{r}$ are 3D unit vectors. The norm of the wave vector, $\mathbf{q}$, is related to the diffusion weighting factor (the b-value), $b=4 \pi^{2} q^{2} \tau$, where $\tau$ is the effective diffusion time.

Diffusion Spectrum Imaging (DSI) reconstruct the EAP. The original DSI protocol 1] measures $S(\mathbf{q})$ on a Cartesian grid inscribed in a sphere of radius 
5, resulting in 515 q-space discrete measurements $S(\mathbf{q})$. Then, a Hanning filter is applied on the raw $S(\mathbf{q})$ 's to reduce truncation errors and boundary artifacts before a simple 3D Fast Fourier Transform (FFT) is applied to recover the EAP at every imaging voxel. Finally, the diffusion orientation distribution function (ODF), $\Psi$, can be extracted by numerically computing the radial integral over $r \in[0,5]$, as $\Psi(\mathbf{u})=\int_{0}^{5} P(r \mathbf{u}) r^{2} d r$. DSI is a long acquisition (approximately $1 \mathrm{~h} 45$ min for a full brain with $2 \mathrm{~mm}$ isotropic voxels). Because diffusion is symmetric, one can cut the acquisition time in half if only one hemi-plane is acquired, resulting in 258 directions 2. DSI has regained popularity in the last years because successful connectomics studies [2] and the human brain connectome project has brought back DSI as the central DWI protocol before fiber tractography.

As any experimental data, DSI data is corrupted by noise, especially because of the large b-values used in the protocol. At these larger b-values, most of the signal is near the noise floor (see Fig. 3). A first challenge is therefore to be able to improve the quality of DSI with denoising algorithms. Another key challenge is the ability to reduce the acquisition time while offering high resolution data required to estimate complex fiber crossings. Hence the goals of this paper are twofold: 1) Denoising of DSI data. 2) Fast DSI acquisition for clinical applications. In particular, can we subsample q-space while keeping good high spectral resolution, i.e. perform DSI at the price of HARDI?

The intuition behind this paper is that the DSI acquisition on 258 points hemi-plane or the full 515 sampling contains redundant information that one can learn to denoise or accelerate DSI acquisition. We propose to use sparse coding to estimate a dictionary of prototypical DSI profiles that well model the structure of the DSI signal. We show that the estimated dictionary of DSI profiles captures the geometry of white matter brain structures and can thus be used to improve the data quality while keeping a small sampling of the q-space. It can be used for 2 things: i) intra-subject studies for denoising purposes and ii) inter-subject studies by using a lower DSI sampling acquisitions to recover the full DSI using a learned dictionary of DSI profiles.

Notations We write vectors in bold, $\mathbf{a} \in \mathbb{R}^{n}$, matrices with capital bold letters, $\mathbf{A} \in \mathbb{R}^{n \times n}$. A scalar $a$ is positive if $a \in \mathbb{R}_{+}$. We denote $\|\mathbf{A}\|_{\text {Fro }}$ the Frobenius norm, $\|\mathbf{A}\|_{\text {Fro }}^{2}=\sum_{i, j=1}^{n} \mathbf{A}_{i j}^{2}$, and $\|\mathbf{A}\|_{1}=\sum_{i, j=1}^{n}\left|\mathbf{A}_{i j}\right|$ the $\ell_{1}$ norm. Column $i$ of a matrix is written $\mathbf{A}^{i}$. If $\mathcal{I}$ is a list of $|\mathcal{I}|$ indices, $\mathbf{A}_{\mathcal{I}}$ is the matrix $\mathbf{A}$ restricted to the rows in $\mathcal{I}$. I stands for the identity matrix. Quantities estimated from the data are written $\widehat{\mathbf{A}}$. A matrix with non-negative elements is denoted $\mathbf{A} \geqslant 0$.

\section{Learning a Dictionary of DSI Profiles with Sparse Coding}

Let $\mathbf{S} \in \mathbb{R}_{+}^{d \times p}$ denote acquired DWI at $p$ voxels over a set of $d$ directions $(d=258$ in the results section below). We consider the following generative model for the DWI data at voxel $i: \mathbf{S}^{i}=\mathbf{D W}^{i}+\mathbf{e}^{i}$, where $\mathbf{D} \in \mathbb{R}_{+}^{d \times k}$ is a dictionary of DSI profiles and $\mathbf{W} \in \mathbb{R}_{+}^{k \times p}$ are the coefficients of the data decomposition over the 
dictionary. The integer $k$ is the size of the dictionary. The noise $\mathbf{e}^{i} \in \mathbb{R}_{+}^{d}$ is known to have a Rician distribution and non-central $\chi$-distribution when using parallel imaging 34. However, for the current contribution, it will be assumed Gaussian with mean $\mu \in \mathbb{R}^{d}$ and diagonal covariance $\boldsymbol{\Sigma}=\operatorname{diag}\left(\left(\sigma_{j}^{2}\right)_{j=1, \ldots, d}\right) \in \mathbb{R}^{d \times d}$. We denote it $\mathbf{e}^{i} \sim \mathcal{N}(\mu, \boldsymbol{\Sigma})$. This modeling assumption is valid for images with SNR above 4 [3], which is the case for the DSI data used in this paper.

The estimation procedure detailed below requires that the additive noise to be Gaussian with unit variance. To meet this constraint, we define the whitened data $\mathbf{S}_{w}^{i}=\boldsymbol{\Sigma}^{-1 / 2}\left(\mathbf{S}^{i}-\mu\right)$ so that $\mathbf{S}_{w}^{i} \sim \mathcal{N}\left(\mathbf{D}_{w} \mathbf{W}^{i}, \mathbf{I}\right)$ where $\mathbf{D}_{w}=\boldsymbol{\Sigma}^{-1 / 2} \mathbf{D}$. In practice, $\mu$ and $\boldsymbol{\Sigma}$ are estimated from voxels with weak diffusion (e.g. the skull).

In order to learn the dictionary $\mathbf{D}$ one needs to set priors on both $\mathbf{D}$ and $\mathbf{W}$. The positivity of DWI data imposes that $\mathbf{D} \geqslant 0$ and $\mathbf{W} \geqslant 0$. The dictionary is a good model for the entire white matter but the data at a given voxel should only be formed by a linear combination of a few DSI profiles: $\mathbf{W}$ should be sparse, i.e. contain many zeros. This leads to the following minimization:

$$
\begin{gathered}
\left(\widehat{\mathbf{D}_{w}}, \widehat{\mathbf{W}}\right)=\underset{\mathbf{D}, \mathbf{W}}{\operatorname{argmin}} \frac{1}{2 d}\left\|\mathbf{S}_{w}-\mathbf{D}_{w} \mathbf{W}\right\|_{\text {Fro }}^{2}+\lambda\|\mathbf{W}\|_{1} \\
\text { s.t. }\left\|\mathbf{D}^{k}\right\|_{2}^{2} \leqslant 1, \mathbf{D} \geqslant 0, \mathbf{W} \geqslant 0
\end{gathered}
$$

The parameter $\lambda$ balances the reconstruction error and the $\ell_{1}$ regularization term which promotes sparse coefficients $\mathbf{W}$. Columns of $\mathbf{D}$ have unit norm to avoid scaling ambiguity. Following [5], we use an online cyclic descent to minimize (2). The estimated dictionary $\widehat{\mathbf{D}}$ is then given by $\widehat{\mathbf{D}}=\boldsymbol{\Sigma}^{1 / 2} \widehat{\mathbf{D}}_{w}$.

Denoising and subsampling. Given a learned dictionary D, one can estimate the coefficients of a decomposition for a new dataset, with possibly less directions than the original dataset used to learn $\mathbf{D}$, i.e. using only a limited set of rows of D. Let us denote $\mathcal{I}$ a list of sampling directions $(|\mathcal{I}| \leqslant d)$. Given a set of whitened subsampled data $\mathbf{S}_{\mathcal{I} w}$, the coefficients $\mathbf{W}$ can be obtained by solving:

$$
\widehat{\mathbf{W}}=\underset{\mathbf{W}}{\operatorname{argmin}} \frac{1}{2|\mathcal{I}|}\left\|\mathbf{S}_{\mathcal{I} w}-\mathbf{D}_{\mathcal{I} w} \mathbf{W}\right\|_{\text {Fro }}^{2}+\delta\|\mathbf{W}\|_{1} \quad \text { s.t. } \mathbf{W} \geqslant 0
$$

where $\delta>0$. The full signal can then be obtained as: $\widehat{\mathbf{S}}=\mathbf{D} \widehat{\mathbf{W}} \in \mathbb{R}^{d \times p}$.

Model selection and choice of parameters. The estimation procedure involves a few parameters, $\lambda$ to learn a dictionary and $\delta$ to estimate the coefficients given the dictionary. In order to tune these parameters we use cross-validation exploiting the symmetry of the signal.

Given half of the directions $\mathcal{H}(\mathrm{d}=258$ directions $)$, a good model should be able to predict the other half by applying a simple symmetry to the data. This observation also allows us to estimate a full dictionary of $2 d-1=515$ directions using only half of the data. The minimization of (2) restricted to the data $\mathbf{S}_{\mathcal{H}}$ gives $\widehat{\mathbf{D}}_{\mathcal{H}} \in \mathbb{R}^{d \times k}$ which leads to $\widehat{\mathbf{D}} \in \mathbb{R}^{2 d-1 \times k}$ by applying a symmetry. 


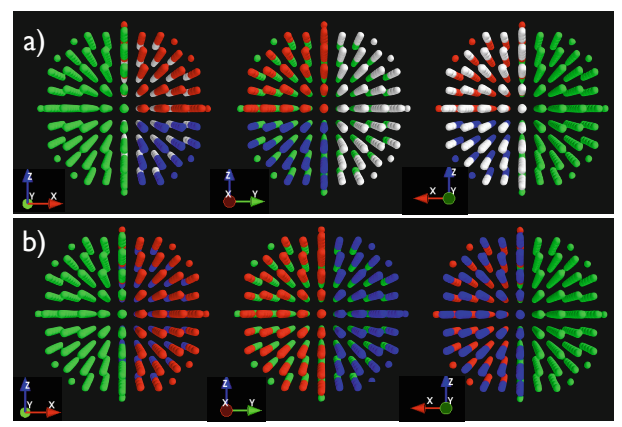

Fig. 1. In green are the 258 directions $\mathcal{H}$ used to estimate a dictionary. a) For intrasubject cross-validation, in red are the directions $\mathcal{L}$ used to estimate the coefficients and in blue the left out directions $\mathcal{T}$ used to evaluate the model parameters. The white directions $\mathcal{V}$ are only used for validation. b) For inter-subject cross-validation the same color code applies. There is no validation set in this case, as the validation data is obtained from the other subject.

In order to assess the quality of the model without overfitting, the model selection involves two other sets of directions: $\mathcal{L}$ to learn $\widehat{\mathbf{W}}$ and $\mathcal{T}$ to test the reconstruction error. The best parameters minimize this error. It is quantified with the average root mean square error (RMSE): RMSE $=\frac{1}{p|\mathcal{T}|}\left\|\mathbf{S}_{\mathcal{T}}-\widehat{\mathbf{D}} \mathcal{\mathcal { T }} \widehat{\mathbf{W}}\right\|_{\text {Fro }}^{2}$. This procedure is a principled way of choosing the parameters. Once they are set, the model estimated can be tested on new DWI $\mathcal{V}$ for validation. As $\mathcal{V}$ has not been used for training, RMSE obtained by different algorithms can be compared.

In the following experiments, the parameter $\lambda$ was set in a range of 5 values $(1,0.1,0.01,0.001)$ and $\delta$ in a logarithmic grid of 15 values between 0 and $1 e^{-6}$. In order to quantify the performance of our method, we use as baseline the solution that consists in applying a simple symmetry to the data, as done classically [2]. We denote $\mathrm{RMSE}_{\text {sym }}$ the error obtained. We report the quality of our solution as a ratio between the two quantities: $\rho_{R M S E}=\mathrm{RMSE}_{\text {sym }} / \mathrm{RMSE}$. A ratio above 1 indicates an improvement with respect to a symmetrization.

The following results involve two setups. An intra-subject denoising procedure and an inter-subject procedure where a dictionary learned on a subject is used to estimate a full resolution DSI dataset for a new subject from only a few DWI. See Fig. 1 for details on $\mathcal{H}, \mathcal{L}, \mathcal{T}$ and finally $\mathcal{V}$ used to estimate $\rho_{R M S E}$.

The data consists of 2 subjects. A standard DSI acquisition mimicking [1] was done with isotropic $2 \mathrm{~mm}$ spatial resolution and $d=515$ DWI were acquired sampling the q-space on a cubic lattice within the sphere of radius 5, $\mathrm{TE} / \mathrm{TR}=147 \mathrm{~ms} / 11.5 \mathrm{~s}, \mathrm{BW}=1680 \mathrm{~Hz} /$ pixel, $96 \times 96$ matrix, and 60 axial slices with a parallel reduction factor of $2, \delta$ and $\Delta$ were 41 and $45 \mathrm{~ms}$, resulting in a maximum q-value of $q_{\max }=70.4 \mathrm{~mm}^{-1}, b_{\max }=6000 \mathrm{~s} / \mathrm{mm}^{2}$. The SNR of the $b=0$ image was 36 and the SNR of the DWI for the $b=960,3360$, and $6000 \mathrm{~s} / \mathrm{mm}^{2}$ datasets were estimated to $12,7.5$, and 6.5 respectively. For the rest of this paper, acquisition of 258 directions with simple symmetry will 
be abbreviated HALF, as opposed to the FULL acquisition. Different symmetry completion procedure are also included for comparison, either using Gaussian smoothing with $\sigma=0.35$ (optimal $\sigma$ in our experiments) and state-of-the-art non-Local (NL) means denoising [6]. Finally, ODFs are computed as described above and visualized as deformed spheres with the radius proportional to $\Psi(\mathbf{u})$.

\section{Results}

Figure 2 shows the ODFs of the learned dictionaries with 100 atoms on both subjects. Atoms are ordered from left to right, starting at the bottom left corner based on the variance they explain on the data. We see that most important atoms are isotropic profiles and several single fiber structures. After approximately 30 atoms, crossing profiles appear. At the end of the dictionary, more complex ODF profiles are also present. This behavior of the learned dictionary is similar if we increase its size $k$.

Intra-subject denoising Table 1 shows how sparse DSI reconstruction is able to accurately reconstruct the un-measured 257 directions. It has lower RMSE than the usual symmetry and, Gaussian and NL means denoising. Increasing the number of atoms in the dictionary only slightly improves the accuracy on subject 2. Moreover, Fig. 3 confirms that denoising, in general, improves the raw DSI data. However, it can be seen that NL means and Gaussian denoising seem to over-smooth and blur the structure of the raw data, as opposed to sparse DSI that appear to denoise but also enhance structure. Finally, Fig. 4 overlays ODFs in a zoom region of this slice, corresponding to the centrum semiovale where corpus callosum (CC) crosses with the corticospinal tract (CT) and superior longitudinal fasciculus (SLF). Single, two and three fiber crossings can be seen. One can appreciate how sparse DSI is able to recover ODF profiles as sharp as the FULL raw and NL means/Gaussian denoised DSI.

Inter-subject undersampling One can push sparse DSI and attempt to perform DSI estimation and ODF reconstruction from undersampled q-space data. The compressed sensing literature teaches us that the "sensing" strategy is crucial for optimal reconstructions. It is beyond the scope of this paper to explore optimal undersampling strategies. Here, we undersampled 1 measurement out of $N$ from the Cartesian direction indices, which preserves a uniform Cartesian sampling.

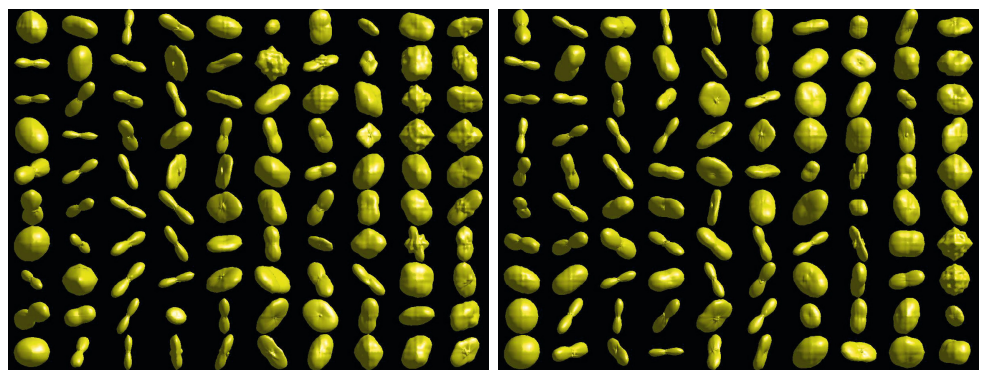

Fig. 2. ODFs computed from the learned dictionaries on the 2 subjects (100 atoms and inter-subject cross-validation). Left (resp. right) is for subject 1 (resp. 2). 
Table 1. Intra-subject denoising. $\rho_{R M S E}$ between simple DSI symmetry, Gaussian smoothing, NL means and sparse DSI denoising. Sparse DSI reconstruction gives the best performance on the validation data.

\begin{tabular}{|l|c|c|c|c|c|c|c|c|}
\hline Methods & $\begin{array}{c}\text { Gaussian } \\
\sigma=0.35\end{array}$ & NL means & \multicolumn{6}{|c|}{ Sparse DSI } \\
& & $k=100$ & $k=169$ & $k=225$ & $k=400$ & $k=900$ & $k=1600$ \\
\hline Subject 1 & 1.16 & 1.19 & 1.31 & 1.31 & 1.31 & 1.31 & 1.31 & 1.31 \\
\hline Subject 2 & 1.13 & 1.16 & 1.28 & 1.25 & 1.23 & 1.30 & 1.30 & 1.29 \\
\hline
\end{tabular}

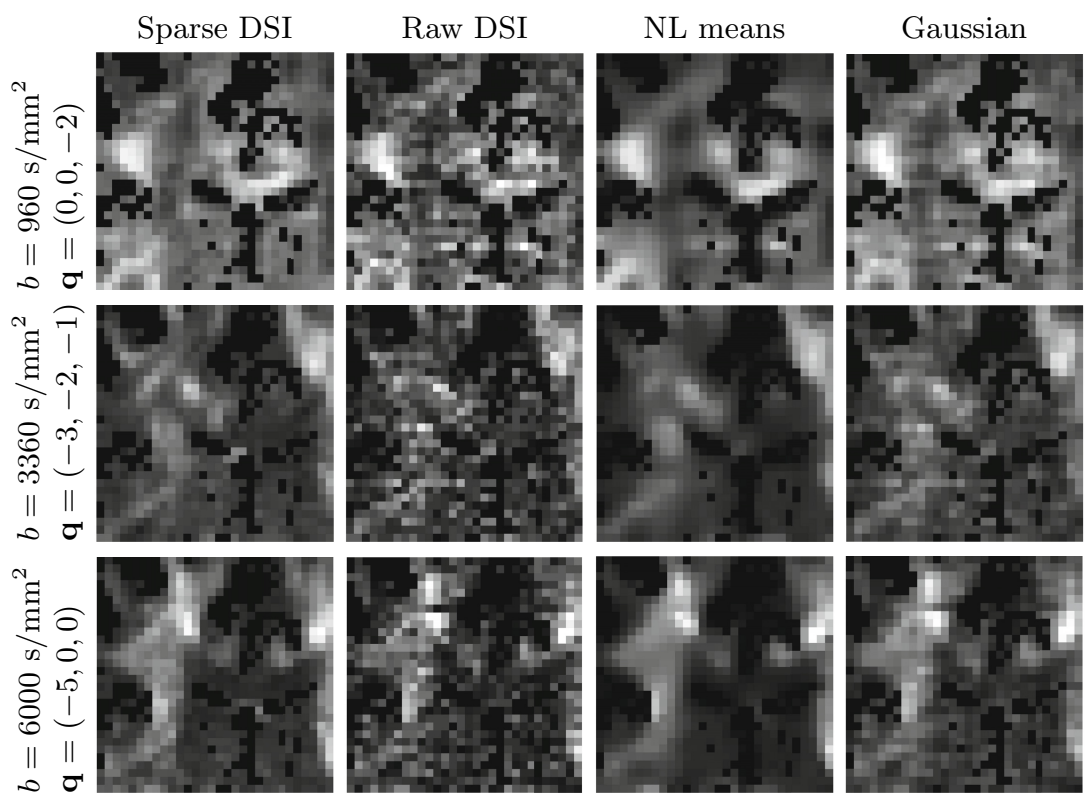

Fig. 3. Denoising the raw data DSI with our sparse DSI technique versus state-of-theart non-local means (NLM) and Gaussian (optimal $\sigma=0.35$ ) denoising.

Figure [5] shows the RMSE ratio between simple HALF DSI with symmetry and sparse DSI as a function of number of measurements. We also show the ODF field in Fig. 6] as a function of number of measurements. First, it is amazing to see that a learned DSI dictionary of a subject can be used to perform undersampled DSI on a different subject. It means that both dictionaries in Fig. 2 look similar and quantitatively yield comparable performances. Of course, as undersampling decreases, the overall field of ODF seems more noisy but the overall RMSE remains acceptable. At a total of 37, 29, and 21 measurements, we become worst than NL means, Gaussian smoothing and simple symmetry DSI in terms of RMSE. On the other hand, we observe that ODF profiles are degraded sooner as a function of undersampling. Note that the structured voxels with single fiber orientation in the CC, CT and SLF are well preserved all the way down to 29 measurements. However, although crossings are found for all undersampling, ODF peaks in crossing areas become less accurate below 58 measurements. 

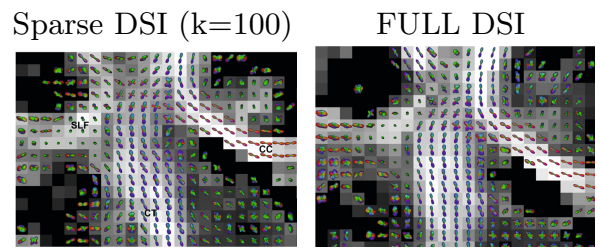

FULL NL means

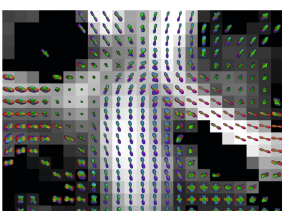

FULL Gauss
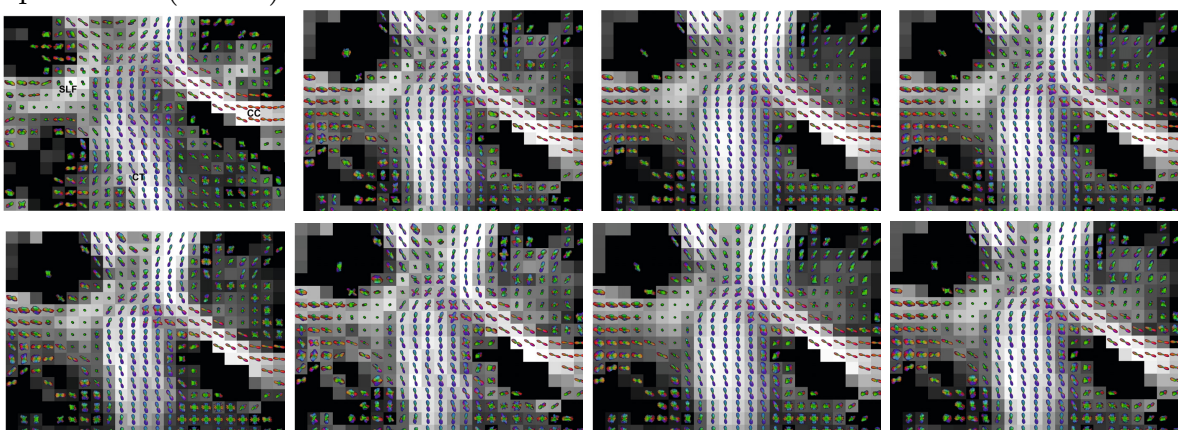

HALF DSI

HALF NL means

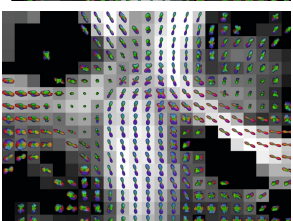

HALF Gauss

Fig. 4. Full $(d=515)$ DSI vs. Half DSI $(d=258)$ with respect to simple symmetry, Gaussian $(\sigma=0.35)$, NL means and sparse DSI denoising of subject 1 (k atoms).

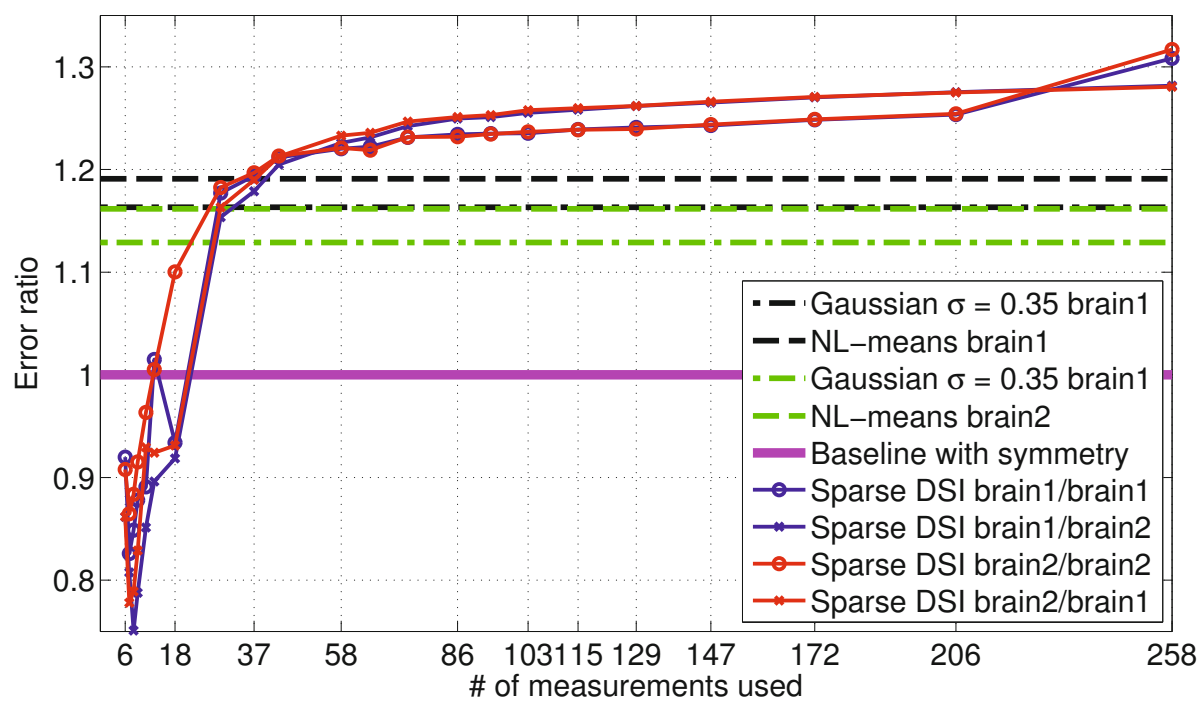

Fig. 5. Reconstruction error ratios for intra and inter-subject settings as a function of the number of measurements ( $\mathrm{k}=100$ atoms). brain1/brain 1 is for the intra-subject case while brain1/brain2 is the inter-subject (Atoms learned on subject 1 and used the estimate the full DSI data of subject 2 using only a few measurements). 

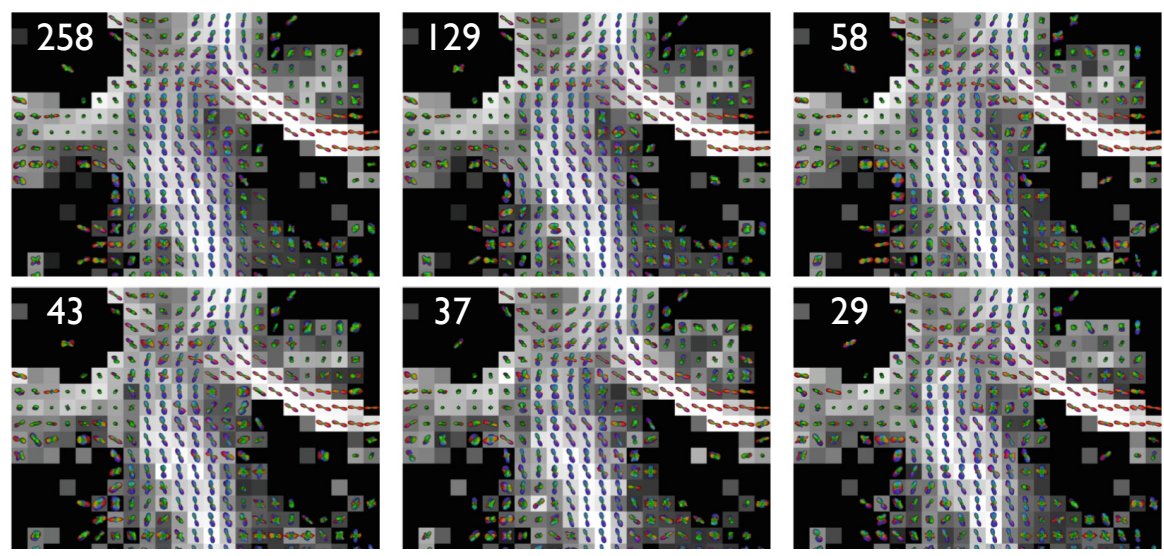

Fig. 6. Undersampled sparse DSI using learned dictionary from subject 1 to reconstruct DSI signal and diffusion ODFs of subject 2. In white is the number of measurements.

\section{Discussion and Conclusion}

Sparse coding applied to DSI data reveals the latent structure of the white matter. Sparse coding is however not compressed sensing (CS), as done for DSI in [7. Although our technique attempts to infer full DSI data from undersampled acquisitions, there is no "sensing" in the technique. The idea is to learn the structure of raw DWI from a full DSI acquisition to either, denoise the DSI data, or use the learned dictionary of DSI profiles to perform undersampled DSI acquisitions and reconstructions. While [7,8] fix a priori the sparse representation of the data (e.g. spherical ridgelets) we propose here to estimate it. The technique proposed is attractive thanks to its little modeling assumptions and its limited number of parameters that can be estimated by cross-validation.

The key benefit of our method is its ability to perform denoising across all the DWI channels jointly, consequently enhancing the image quality in particular for noisy high b-values. While the technique of [10] uses DW images within a certain cone around the DW image being denoised, we propose to estimate the underlying structure from all directions and b-values. This is made possible by a proper whitening of the data in order to combine in the estimation multiple images corrupted by different noise levels.

Results have showed that with just half of the data (258 DWI), we can better predict the other 257 DWI than the classic symmetry procedure. This statement also holds even if we use as little as 40 q-space measurements. Our sparse DSI technique performs better than symmetrizing, Gaussian denoising or state-ofthe-art NL means. Finally, beyond denoising, we have showed that learning the dictionary from one subject can be used to reconstruct full DSI dataset from an undersampled acquisition of a different subject. From now on, we could acquire around 40 measurements on new subjects and use the learned dictionaries to 
reconstruct a full DSI data. Hence, we can have fast acquisitions to obtain high resolution DSI data. Therefore, DSI can be done at the price of HARDI!

Future work will be dedicated at optimizing the dictionary learning to enhance ODF reconstruction and also to find optimal sub-sampling strategies.

\section{References}

1. Wedeen, V.J., Hagmann, P., Tseng, W.Y.I., Reese, T.G., Weisskoff, R.M.: Mapping complex tissue architecture with diffusion spectrum magnetic resonance imaging. Magnetic Resonance in Medicine 54(6), 1377-1386 (2005)

2. Hagmann, P., Cammoun, L., Gigandet, X., Meuli, R., Honey, C.J., Wedeen, V.J., Sporns, O.: Mapping the structural core of human cerebral cortex. PLoS Biology $6(7)$, e159 (2008)

3. Sijbers, J., den Dekker, A.J., Audekerke, J.V., Verhoye, M., Dyck, D.V.: Estimation of the noise in magnitude MR images. Magnetic Resonance Imaging 16(1), 87-90 (1998)

4. Koay, C.G., Özarslan, E., Basser, P.J.: A signal transformational framework for breaking the noise floor and its applications in MRI. Journal of Magnetic Resonance 197(2), 108-119 (2009)

5. Mairal, J., Bach, F., Ponce, J., Sapiro, G.: Online learning for matrix factorization and sparse coding. Journal of Machine Learning Research 11, 19 (2010)

6. Coupé, P., Manjón, J.V., Gedamu, E., Arnold, D., Robles, M., Collins, D.L.: Robust rician noise estimation for MR images. Medical Image Analysis 14(4), 483-493 (2010)

7. Menzel, M.I., Tan, E.T., Khare, K., Sperl, J.I., King, K.F., Tao, X., Hardy, C.J., Marinelli, L.: Accelerated diffusion spectrum imaging in the human brain using compressed sensing. Magnetic Resonance in Medicine 66(5), 1226-1233 (2011)

8. Landman, B.A., Bogovic, J.A., Wan, H., ElShahaby, F.E.Z., Bazin, P.L., Prince, J.L.: Resolution of crossing fibers with constrained compressed sensing using diffusion tensor MRI. NeuroImage 59(3), 2175-2186 (2012)

9. Michailovich, O., Rathi, Y., Dolui, S.: Spatially regularized compressed sensing for high angular resolution diffusion imaging. IEEE Transactions on Medical Imaging 30(5), 1100-1115 (2011)

10. Tristán-Vega, A., Aja-Fernández, S.: DWI filtering using joint information for DTI and HARDI. Medical Image Analysis 14(2), 205-218 (2010) 